\title{
Energy Efficient Adaptive Mobility of MAC Protocol using Sleep and Wake Cycling in Wireless Sensor Network
}

\author{
Jagdish Y. Kapadnis \\ Research Scholar \\ Department of Computer science and Engineering. \\ LNCT, Indore, INDIA
}

\author{
Pawan Patidar \\ Assistant Professor \\ Department of Computer science and Engineering. \\ LNCT, Indore, INDIA
}

\begin{abstract}
Energy efficiency has been an important concern in wireless sensor networks where Medium Access Control (MAC) protocol plays an important role. MAC protocols proposed for wireless sensor networks assume static sensor nodes but nodes know their locations, and they sleep-wake cycle, waking up periodically but not synchronously, which usually causes up gradation in network performance in scenarios involving mobile sensors. When a node has a packet to forward to the sink, there is a trade-off between how long this node waits for a suitable neighbor to wake up and the progress the packet makes towards the sink once it is forwarded to this neighbor .In this paper, it is introduce a mobility aware and energy efficient medium access protocol with sleep and wake up cycle (shortly abbreviated as MMAC-SW) for mobile wireless sensor networks. Our MMAC-SW is based on a hybrid scheme of TDMA and CSMA that informs sensor nodes when to wakeup or when to go to sleep to save energy. Through computer simulations, it is evaluate the performance of the MMAC-SW and compare it against the MMAC protocol. Simulation results will show that MMAC-SW better performs than MMAC in terms of energy consumption, packet delivery ratio and average packet delay.
\end{abstract}

Keywords: energy efficient, MAC protocol, mobility handling, sleep and wake cycling.

\section{INTRODUCTION}

A wireless sensor network (WSN) is a network of self organizing Low powered devices having sensing and communication capabilities. These small and inexpensive devices are battery powered, thus sensor nodes must be energy efficient [4]. In general, routing protocols are classified into three i.e. data-centric, hierarchical and location-based protocols. Data-centric protocols are usually query-based and work based on the requested data. Hierarchical approaches aim at clustering the nodes so that cluster heads are capable of doing data aggregation and decreasing data overhead for conserving energy. Location-based protocols utilize the location of data to relay the information to the required areas. As developments of these micro-electro-mechanical systems, digital electronics, and wireless communications technologies have led to create low cost, low power and multifunctional sensor devices [3], in such WSNs, typically the nodes rely on batteries, or energy harvested from their surroundings, and, hence, need to be extremely parsimonious in their use of energy. In order to conserve energy, the nodes operate in sleep-wake cycles. In such WSNs, typically the nodes rely on batteries, or energy harvested from their surroundings, and, hence, need to be extremely parsimonious in their use of energy. In order to conserve energy, the nodes operate in sleep-wake cycles, when a node wakes up it performs sensing, and also can assist in forwarding any alarm packets towards the sink.

In each cluster, one of the members will be selected as the cluster head to control and manage the data exchanged between nodes in the cluster and report the collected data to a BS through one or more clusters. Each cluster head maintains neighbor table that includes information about its member nodes and adjacent cluster heads. In addition, other members will be synchronized with their cluster head. There are numerous advantages of MMAC-SW using sleep and wake up cycle over the static WSNs. In particular, it offers:

i) Dynamic network coverage [1], by reaching areas that have not been adequately sampled. ii) Data routing repair, by replacing failed routing nodes with their adequate node in the network. iii) Data mulling, by collecting and disseminating data/reading from stationary nodes out of range. iv) Stage data stream processing, by conducting in-network processing of continuous and ad hoc queries. v) User access points, by enabling connection to handheld and other mobile devices that are out of range from the communication infrastructure.

Cluster heads gather information about their clusters and later transmit the information to the sink node. Here, we should aware about the data delivery from cluster heads to the BS as each cluster head needs to transfer large amount of data. In this area, some intermediate nodes act as bridges between cluster heads and the BS which should frequently transfer large amount of data. However, performing this task gradually exhausts intermediate nodes energy and makes them die sooner than other nodes. Therefore, loosing these bridges leads to disconnection between the nodes and the BS and reduces the network lifetime significantly.

In this paper it is propose that an efficient data routing approach which reduces the energy consumption of the network and provides reliable data delivery to the BS Mobility prediction through the use of the first order auto aggressive moving average model [14] is used to dynamically adjust the frame size and control the channel access in an efficient way according to the mobility conditions. We have designed and implemented the MMAC-SW protocol in event network simulator, where many simulation experiments are carried out to evaluate the performance of MMAC-SW and compared it with MMAC [14]. The rest of the paper is organized as follows. We present and discuss some related works in section II. Section III Describes the MMAC-SW protocol, in section IV describes what will be the system model of the protocol. In section V Describes the Data transfer phase performance is studied through intensive 
simulation experiments. Finally the paper is concluding in section VI.

\section{RELATED WORKS}

According to channel access policies, the MAC protocols in WSN are generally categorized into three classes: contentionbased, TDMA-based, and hybrid MAC layer protocols. Contention- based MAC is based on the idea that when one node needs to send data it will compete for wireless channel. It is therefore simple to implement and has good scalability. TDMA based MAC protocols have a natural advantage of collision-free medium access. However, they suffer heavily from problems like clock synchronization, channel underutilization and fixed time-slot assignments. Therefore, TDMAbased MAC [9] has poor performance at varying traffic loads. Hybrid MAC [10] combines the advantages of contention-based MAC with that of TDMA-based MAC thus it has good adaptability to traffic variation. However, hybrid MAC protocols are usually very complex and need complicated scheduling algorithms which waste much energy. Therefore, in this paper mainly focus on contention-based MAC protocols. Since the collision and control overhead tradeoff also exists in other type of MAC protocols but has been less addressed for multiple coexisting applications currently, we will consider extending our method to TDMA and Hybrid MAC later for future investigation. Contention-based MAC is generally based on CSMA/CA or CSMA. It could be classified into synchronous and asynchronous modes to compete for the channel before transmitting data. Typical synchronized contention-based MAC protocols include [4] S-MAC [2] adopts a periodic listen-sleep cycle to reduce idle listening and employs RTS/CTS/DATA/ACK handshake mechanism similar to IEEE 802.11 to avoid collisions and overhearing. However, the duration of the active and sleep period is fixed, which result in increasing idle listening under low traffic load. T-MAC [3] enhances the poor performance produced by S-MAC under variable traffic load. It introduces an adaptive duty cycle, all messages are transmitted in variable length bursts and the lengths of bursts are dynamically determined. Due to the periodical listen-sleep cycles adopted in [2] and [3], both SMAC and TMAC are applicable for periodical data gathering applications.

PS-MAC [4] was proposed in which each node determines 'listen' or 'sleep' pseudo-randomly based on its own prewakeup probability and pre-wakeup probabilities of its neighbor nodes in each time slot. However, the optimal pre-wakeup probability of each node based on the congestion level of each node and the network topology has not been studied. B-MAC [5] uses long-length preambles to reduce duty cycle and minimize idle listening time, which results in the energy waste in sender and receiver, and overhearing in neighbor nodes of the sender will arise. Due to long preambles and synchronization, B-MAC is applicable for non-real time application. Wise MAC [6] dynamically adjusts the length of preambles by piggybacking the wake-up time in latest ACK packet which shortens the length of preambles. However, Wise MAC uses non-persistent CSMA to reduce idle listening so the hidden terminal problem still exists. It is suitable for query-driven application. X-MAC [7] further shortens the length of preambles by using strobe preambles. However, when the traffic load varies with time, energy waste on idle listening, collisions and overhearing still exists. It is usually adopted in event-driven application.

\section{MMAC-SW PROTOCOL}

The synchronous protocols, due to the negotiation of the wakeup time schedule with the neighbors during each duty cycle reduce the idle listening energy consumption. For example in S-MAC [4], as shown in Fig. 1 each node operates a periodic synchronized schedule for duty cycle composed of LISTEN period and SLEEP period each period is located at only one time on one duty cycle. LISTEN period i.e. the wakeup period, is divided into SYNC and DATA periods. SYNC period is for the synchronization of the duty cycled schedule and DATA period is for actual data communications including Request-To-Send (RTS) and Clear-To-Send (CTS) signals that can help avoid channel collision problems. All of the nodes sleep in the SLEEP period. This example shows that the synchronous MAC protocols based on the duty cycled mechanism are more energy efficient than traditional schemes due to the reduction of both the unnecessarily idle listening time and channel collision probability by the synchronization and RTS/CTS signals respectively. On the other hand, during high traffic condition the successful delivery ratio per duty cycle is limited. In S-MAC the wakeup period ratio of each duty cycle is fixed and it leads to the limited actual delivery throughput. As a result, the synchronous schemes can be inefficient in terms of the energy efficiency and communication delay in high traffic condition. Contrarily, in low traffic condition, fixed periodic wakeup period leads to unnecessary energy consumption. Hence, it is needed that wakeup period management method has to consider the traffic condition.

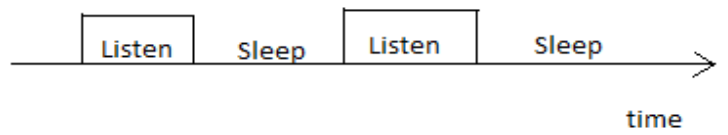

Figure 1. Node operates a periodic synchronized schedule for duty cycle composed of LISTEN period and SLEEP period

The general asynchronous WSN MAC protocols operate independent duty cycle schedule without performing the wakeup time synchronization. In these MACs, due to the lack of information regarding the neighbor's wakeup time, a sender has to guarantee the receiver's wakeup time. In order to accomplish this, many asynchronous protocols based on duty cycled mechanism employed the preamble sampling scheme called Low Power Listening (LPL) to ensure the wakeup condition of the peer node. These protocols tried to enhance the energy efficiency through removing the periodic control frames.

\section{SYSTEM MODEL}

\subsection{Node Deployment}

$\mathrm{N}$ identical sensor nodes are uniformly deployed in the region $\mathrm{R}$. We take $\mathrm{N}$ to be a Poisson random variable of rate $\beta$ where is the node density. Let $x i, i=1,2, \ldots, N$, be the locations of the nodes. Additional source and sink nodes are placed at fixed locations $\mathrm{X} 0=(0,0)$ and $\mathrm{XN}+1=(\mathrm{L}, \mathrm{L})$ respectively. Thus including the source and sink nodes, there are a total of $\mathrm{N}+2$ nodes in the disk. rc is the communication range of each node. Two nodes $\mathrm{i}$ and $\mathrm{j}$ are called neighbors if and only if $|\mathrm{xi}-\mathrm{xj}|<$ rc. The distance between node $\mathrm{i}$ and sink $(\mathrm{N}+1)$ is $\mathrm{Li} \mid \mathrm{XN}+1-$ $\mathrm{Xi}$. 


\subsection{The Adaptable Wakeup Period (AWP)}

In duty cycle based WSN MAC protocols, the important parameters such as delivery delay and energy consumption are influenced by the duty cycle length and wakeup period [3, 4 and 19]. If the allocation of the wakeup period increases, the successful delivery ratio becomes high and retransmission counts decrease, however, the energy performance is going to be inefficient. Contrarily, for a short wakeup period in the same duty cycle, the successful delivery ratio and retransmission counts are worse, however, the energy performance is going to be more efficient. The significant factor of this trade-off issue depends upon the management policy determining the allocation ratio of wakeup period.

In order to address this issue, AWP considers the dynamic network condition gathered from the channel information of the physical layer, and varies the wakeup period: we call this proposed scheme as AWP (Adaptable Wakeup Period). The proposed scheme focuses on the receiving operation. The adjustment of the wakeup period is performed at the receiver, considering the traffic condition within a one hop range. Since traffic condition varies, we use the Clear Channel Assessment (CCA) value to measure the traffic amount. In order to detect the exact radio channel conditions, we monitor and count the CCA value during the performance of the duty cycled mechanism: most radio transceivers provide the CCA value through some registers. In the asynchronous protocols, the CCA value is generally used for CSMA mechanism. When a sender tries to send a data packet to a receiver, the sender checks the CCA value after waiting for some duration which is the random back-off delay time, in the wakeup period. It then sends the actual data packet to the receiver if the CCA indicates an idle state in the radio channel. Otherwise, if the CCA indicates a busy state in the radio channel, the sender re-enters the above delay process. In the asynchronous protocols, the proposed scheme does not need the extra CCA counting process. However, in the synchronous protocols or non-CSMA based asynchronous protocols, the extra process of monitoring and counting the CCA is required in the proposed scheme. Therefore, we recommend periodic CCA measurement from the physical layer. We refer to it as CCA sampling.

\subsection{The Sleep-Wake Process}

To conserve energy, each node performs periodic sleep-wake cycling. The sleep-wake times of the nodes are not synchronized. Since we are interested in studying the delay incurred in routing due to sleep-wake cycling alone, we neglect the transmission delay, propagation delay and other overhead delays. This means that if node $i$ has a packet to transmit to its neighboring node $j$, then $i$ can transmit immediately at the instant $\mathrm{j}$ wakes up. We model this by taking the time for which a node stays awake to be zero. More formally, let $\mathrm{Ti}, \mathrm{i}=1,2$, $\ldots \mathrm{N}+1$ be i.id. Random variables which are uniform on $[0, \mathrm{~T}]$, where $\mathrm{T}$ is the period of the sleep wake cycle. Then node $\mathrm{i}$ wakes up at the periodic instants $\mathrm{KT}+\mathrm{Ti}, \mathrm{K}>0$. We define the waiting time for $i$ to wake up at time $t$ as,

$\mathrm{Wi}(\mathrm{t})=\inf \{\mathrm{KT}+\mathrm{Ti}>\mathrm{t}: \mathrm{k}>0\}-\mathrm{t}$.

\subsection{Forwarding Rules and Assumptions}

Forwarding rules dictate the actions a node can take when it has to transmit. We are interested in decentralized policies where a node can take decisions only by observing the activities in its neighborhood (i.e., the disk of radius rc) centered around the node of interest). In this regard we impose some restrictions on the network.
Traffic Model: There is a single packet in the network which is to be routed from the source to sink. At time 0 , the packet is given to the source and the routing process begins. The nodes which get the packet for forwarding are called relay nodes. The packet traverses a sequence of relay nodes to eventually reach the sink, at which time the routing ends. Thus there is a single flow and further the flow consists of only one packet. This set up is reasonable, because in sensor networks we can assume that the events are sufficiently separated in time and/or location so that the flows due to two events do not intersect. To avoid multiple packet transmission by different nodes detecting the same event, the nodes can resolve among themselves to select one node (say the one closest to the sink), which can then transmit. Further, the information about an event comprises its location, and possibly target classification data, which along with some control bits can be easily incorporated in a single packet. This justifies the idea to study the performance of a single packet alone.

Forwarding Set: Each node knows its location and the location of the sink. The forwarding set of a node is the set of its neighbors that are closer to the sink then itself. A relay node considers forwarding the packet only to a node in its forwarding set. Each node knows the number of neighbors in its forwarding set, but is not aware of their locations and wake times. While in this paper we assume that each node knows the number of nodes in its forwarding set, it would be desirable to develop forwarding algorithms that do not require even this knowledge.

\subsection{Data Transfer Phase}

After the compilation of the clustering phase and the $\mathrm{CHs}$ are advertised, the data transfer phase begins. Data transfer in MMAC-SW is based on frames and the CHs control the frames. The $\mathrm{CH}$ is responsible for controlling the channel access between sensor nodes within the cluster and collects data from them. The frames are handled during multiple phases using a hybrid scheme of CSMA and TDMA. Each frame is composed of two slots (see Fig. 1): mini slot and a dynamic normal slot. The Mini-slot is used to transmit and receive control signals, and consists of three parts; Frame Synchronization (SYNC), Random Access, and Receive Scheduling. The Normal slot is used by sensor nodes to report their data to the $\mathrm{CH}$. The frame length is made dynamic to make the protocol sensitive to mobility and traffic conditions (i.e. the number of time slots is increased or decreased according to the number of nodes that have data to send).

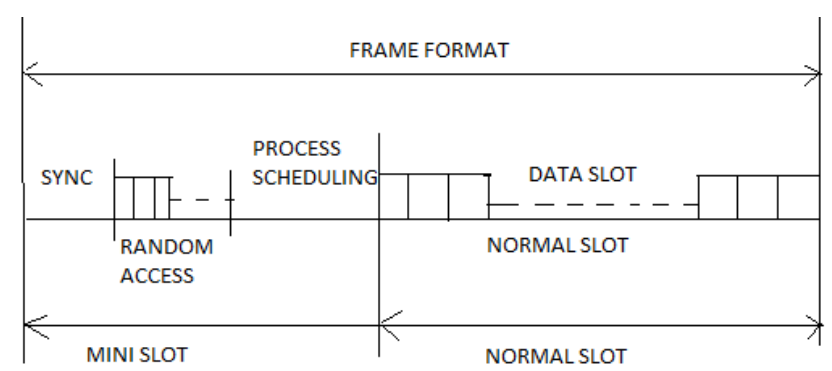

Figure 2 frame format Structure

MMAC-SW protocol handles the channel access through the following four phases:

Schedule Calculation and Distribution, and Data Transfer. Nodes that have data to send should content for the channel during the Request/Update/Join phase and send their requests to the $\mathrm{CH}$. As well those nodes which want to join or leave 
to/from the cluster should send requests during this phase. Then, sensor nodes use the TDMA slots calculated and distributed by the $\mathrm{CH}$ to send their data during the data transfer phase to CHs. Sensor nodes that have no data to transmit go to sleep directly after the end of the mini-slot. More details are given below about the operation of the MEMAC protocol in different phases:

Synchronization phase: At the beginning of each frame, the head node broadcasts a SYNC message to all sensor nodes all sensor nodes should be in receive mode during this phase to be able to capture the SYNC message. The SYNC message contains synchronization information for the packet Transmission.

Request/Leave/Join phase: During this phase, sensor nodes that have data to transmit content for the channel in order to acquire the access to send its request to the $\mathrm{CH}$. The contention period should be long enough to enable all nodes that have data to send to pass their requests to the $\mathrm{CH}$. As well, those nodes which are expected to leave or join the cluster should inform the $\mathrm{CH}$ by sending a leave or join message.

Schedule Calculation and Distribution phase: In this phase the $\mathrm{CH}$ calculates the schedule and broadcasts it to the other nodes within the cluster. The schedule contains those nodes which have data to send only. Nodes that want to leave or join the cluster are not considered in the current schedule. Thus, the frame length is adjusted according to the number of request, leave, and join messages. If the number of request messages are greater than the number of join/leave messages, then the frame length is increased otherwise the frame length is reduced. The frame adjustment algorithm of the MEMAC protocol is an improved version of the mobility adaptive algorithm presented in [13], the algorithm is as follow:

1. For all nodes within the cluster, calculate the predicted states using the AR-1 model.

2. For all nodes in the cluster, calculate the average estimated location.

3. Using the above information construct the set of joining ' $\mathrm{J}$ ' and leaving 'L' nodes.

4. Count the request messages and construct a set of nodes which have a data to send ' $\mathrm{R}$ '.

5. If a node $\mathrm{n}$ is a member of the set of joining or leaving nodes, do not consider $\mathrm{n}$ in the schedule.

6. If the number of members in set ' $R$ ' are greater than the number of members in both sets of ' $J$ ' and ' $L$ ', then increase the frame length, otherwise reduce the frame length.

7. Adjust the frame normal slot and the random access period in the frame structure according to the new frame time.

Finally, the $\mathrm{CH}$ broadcasts the schedule packet to all sensor nodes that contains the TDMA slots for the subsequent phase "data transfer phase". In this phase all sensor nodes should be in receive mode.

Data Transfer phase: In this phase, sensor nodes use the TDMA slots to transmit their data to the $\mathrm{CH}$ or to communicate with their neighbors. All sensor nodes that have no traffic to transmit or receive should turn their radios transceivers off and enter sleep mode. Once data are reported to $\mathrm{CHs}$, the BS collects data from CHs. A dynamic TDMA scheme is used to allow $\mathrm{CHs}$ to access the channel and report their data to the BS. The BS is responsible of calculating and distributing time slots to $\mathrm{CHs}$. We assume that all $\mathrm{CHs}$ have data to report to the BS.
As a result, the random access period is removed, and the frame becomes as shown in Fig. 3.

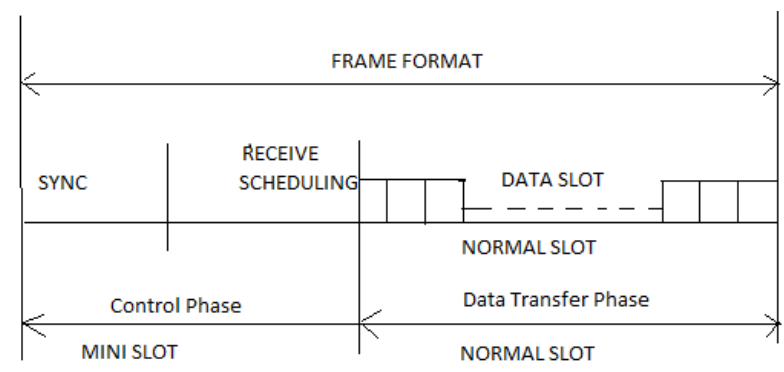

Figure 3 Frame used to send data from CHs-2-BS

\section{PERFORMANCE ANALYSIS}

To study and evaluate the performance of the MMAC-SW protocol and compare it with MMAC protocol, we are created the wireless Sensor network Simulator framework [12]. The simulator contains a model of the wireless sensor node [16]. Furthermore, the simulator has the limited no of nodes. According to simulator we are form a one cluster head according to layers of the WSN protocol stack. The Energy consumption in the simulator is based on the amount of energy the radio consumes. The consumed energy by the CPU as a result of the protocol execution is not taken into account. The motivation behind using Sensor Simulator is that it enables users to easily modify the sensor network and to implement large scale networks. Furthermore, it executes at least an order of magnitude faster than NS-2 and makes more efficient use of the available memory [24]. In this section we investigate the performance of the proposed protocol MMAC-SW in a clustered network topology, and compare it against MMAC protocol. The performance metrics used in the evaluation of MMAC-SW will be the average energy consumption, average packet delivery ratio and average delay.

\subsection{Average Energy Consumption}

Energy efficiency is the most important performance metric in wireless sensor network. Fig. 4 (round1) \& Fig. 5 (round 2) show the energy consumption of MMAC-SW protocol under variable load. Here the average mobility is set to 0 , i.e. static network. Results show that there is a slight improvement in energy consumption of MMAC-SW.

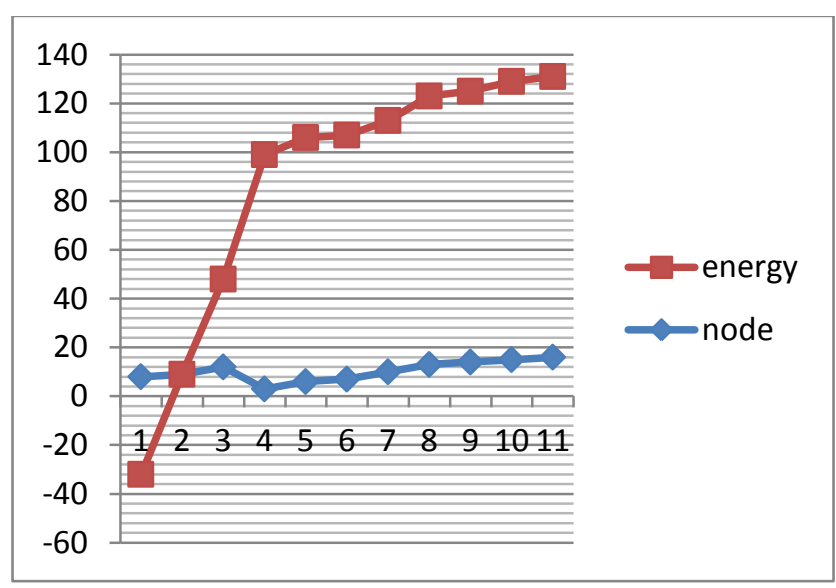

Fig 4: Average energy consumption in round 1 


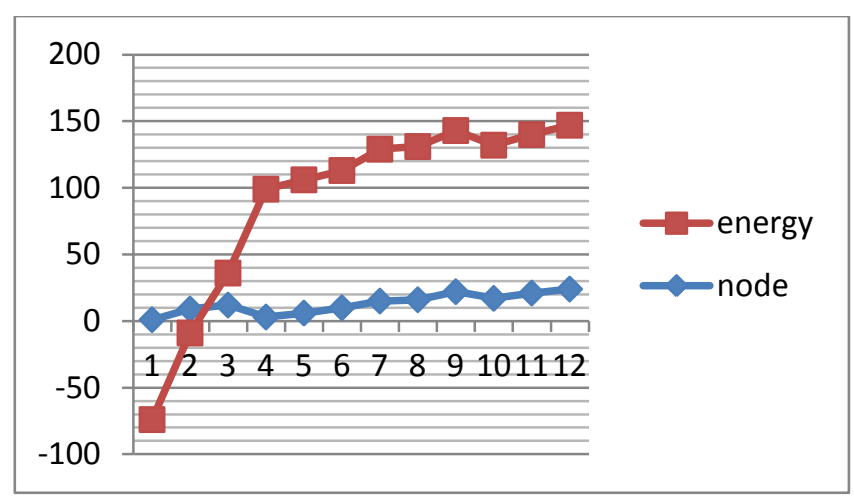

Fig 5: Average energy consumption in round 2

\subsection{Average Packet Delivery Ratio}

Fig.6 (round 1) and Fig. 7(round 2) show the average percentage of data packets successfully delivered to the BS. As the mobility rate increases, the packet delivery ratio for MMAC decreases significantly, while MMAC-SW exhibits a minimal decrease.

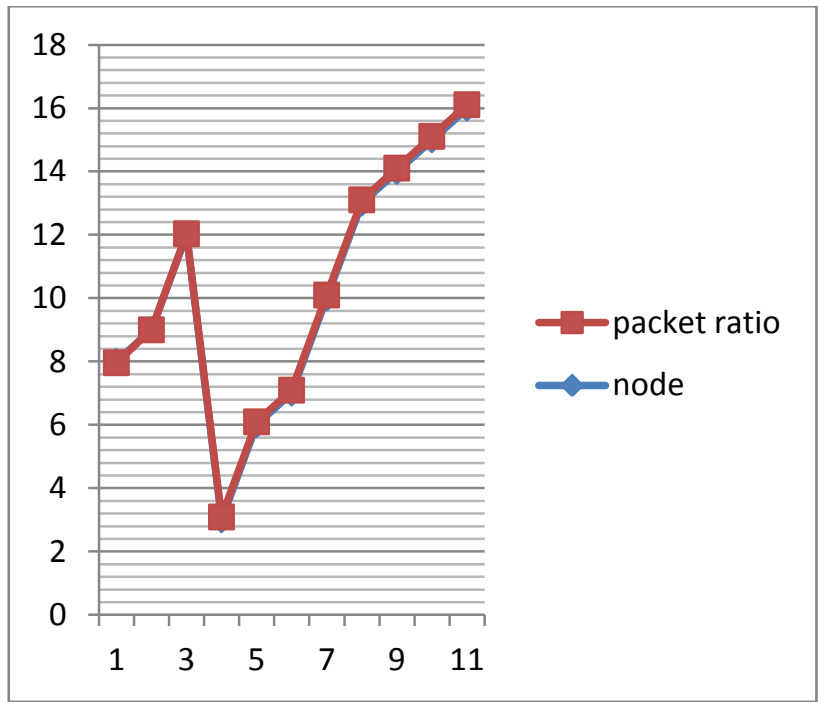

Fig.6 average packet delivery from base station in round 1

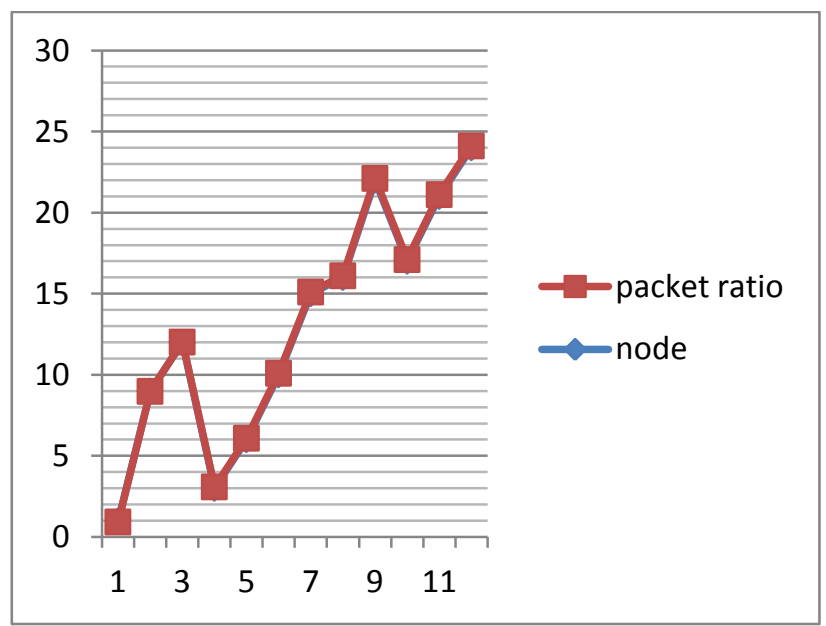

Fig.7 average packet delivery from base station in round 2

\subsection{Average Delay (energy consumption and packet delivery)}

Fig.8 (round 1) and Fig. 9(round 2) shows the average packet delay for MMAC-SW protocol. In this experiment, we vary the traffic load by changing the packet generation time on the source node. It includes average energy consumption and average packet delivery ratio both have high average packet delay, but still MMAC-SW slightly shows better performance.

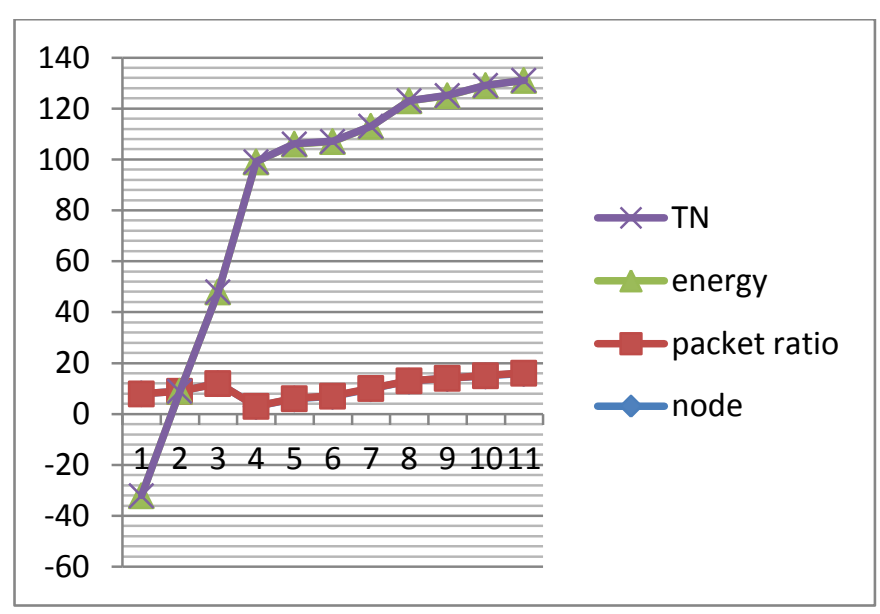

Fig 8: Average delay in round 1

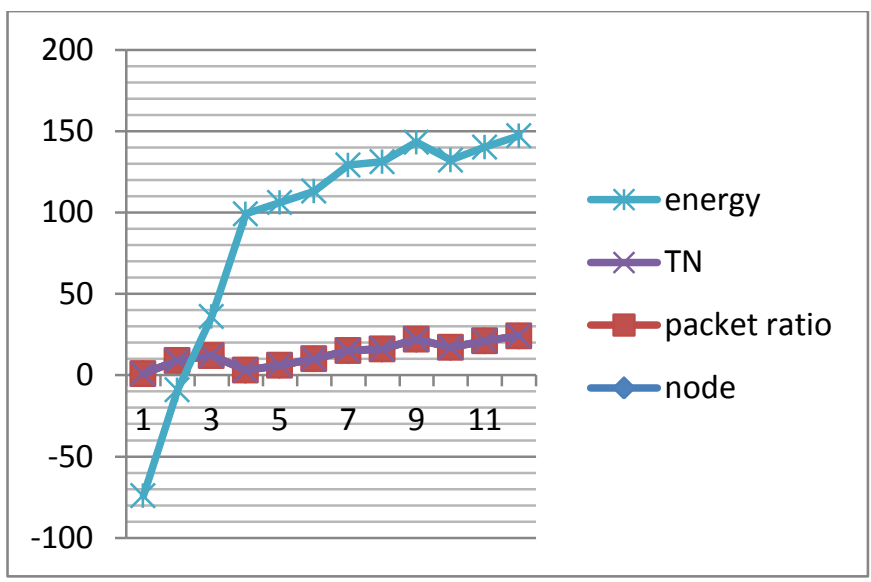

Fig 9: Average delay in round 2

\section{CONCLUSION and FUTURE WORK}

The evolution of stationary WSNs in conjunction with the advances made by the distributed robotics and low power embedded systems communities have led to a new class of Mobile Wireless Sensor Networks (MSNs). In addition to the energy constraints and processing power limitations, mobility in MSN adds a new design dimension. Most of the proposed MAC protocols for WSN networks are designed assuming that sensor nodes are stationary. This assumption no longer valid for MSNs, Therefore designing a mobility aware MAC protocols becomes more and more important. In this paper, we presented MMAC-SW protocol - an adaptive mobility aware and energy efficient MAC protocol for MSNs using sleep and wake up cycles. MMAC-SW combines the benefits of contention based and scheduled based protocols to achieve a significant amount 
of energy savings. MMAC-SW adjusts the frame length according to mobility information of the sensor nodes and the number of nodes that have data to send, this avoids wasting slots by excluding the nodes which are expected to leave or join the cluster and those nodes which have no data to transmit from the TDMA schedule, and to switch nodes to sleep mode when they are not included in the communication process. Through extensive simulation experiments, we are proposed our protocol and compared it against MMAC protocol. Simulation results will demonstrate that our MMAC-SW protocol performs the energy efficient in terms of average energy consumption, average delivery ratio, and average packet delay.

\section{REFERENCES}

[1] Bashir Yahya, Jalel Ben-Othman, "An Adaptive Mobility Aware and Energy Efficient Mac Protocol for wireless Sensor Networks", 978-1-4244-4671-1/09/\$25.00 @2009 IEEE

[2] J. Kim, X. Lin, and N. Shroff, "Optimal Anycast Technique for Delay-Sensitive Energy-Constrained Asynchronous Sensor Networks," in INFOCOM 2009. The 28th Conference on Computer Communications, IEEE, April 2009, pp. 612-620.

[3] Bergbreiter, S. and Pister, K.SJ, "CotsBots: An Off-theShelf Platform for Distributed Robotics," In the Proceeding of the IEEE International Conference on Intelligent Robots and Systems, Las Vegas, NV, 2003.

[4] Dantu K., Rahimi M.H., Shah 1-1., Babel S., Dhariwal A., and Sukhatme G.S., "Robornote: Enabling mobility in sensor networks", In ACM Int. Conf. on Information Processing in Sensor Networks - SPOTS, 2005.

[5] Allred 1, Hasan A.B., Panichsakul S., Pisano B., Gray 1'., Huang J-I-I., I-Ian R., Lawrence D., and Mohseni K., "SensorFlock An Airborne Wireless Sensor Network of Micro-Air Vehicles", In ACM Int. Conf. on Embedded Networked Sensor Systems, 2007.

[6] NittelS, TrigoniN, Ferentinos K., Neville F., Nural A., and Pettigrew N., "A drift-tolerant model for data management in ocean sensor networks", In ACM Workshop on Data Engineering for Wireless and Mobile Access, 2007.

[7] Hull B., Bychkovsky V., Chen K., Goraczko M., Miu A., Shih E., Zhang Y., Balakrishnan H., and Madden S., "CarTel: A Distributed Mobile Sensor Computing System", In ACM Int. Conf. on Embedded Networked Sensor Systems, 2006.

[8] Eriksson, Girod, L., Hull, B., Newton, R., Madden, S. and Balakrishnan H., "The Pothole Patrol: Using a Mobile Sensor Network for Road Surface Monitoring", In ACM Int. Conf. on Mobile Systems, Applications And Services 2008.

[9] D. Zeinalipour-Yazti, H. Papadakis, C. Georgiou, M.D. Dikaiakos, "Mobile Sensor Network Data Management", Parallel Processing Letters Journal (PPL), September 2008.
[10] Lingxuan $\mathrm{Hu}$ and David Evans, "Localization for Mobile Sensor Networks", Tenth Annual International Conference on Mobile Computing and Networking (ACM Mobi Com 2004). 26 September - 1 October 2004.

[11] M. Zhang, X. Du, and K. Nygard, "Improving Coverage Performance in Sensor Networks by Using Mobile Sensors," Proc. IEEE Military Comm. Conf. (MILCOM '05), pp. 3335-3341,2005.

[12] Bashir Yahya, Jalel Ben-Othman, "Energy Efficient MAC Protocols in Wireless Sensor Networks", will appear in Wiley series on Wireless Communications and Mobile Computing, 2009.

[13] Bashir Yahya, Jalel Ben-Othman,"A scalable and energyefficient hybrid-based MAC protocol for wireless sensor networks", in the Proceedings of the 3rd ACM workshop on Performance monitoring and measurement of heterogeneous wireless an wired networks (PM2HW2N 2008), 67-71

[14] H. Takagi and L. Kleinrock, "Optimal Transmission Ranges for Randomly Distributed Packet Radio Terminals," Communications, IEEE Transactions on. [legacy, pre - 1988], vol. 32, no. 3, pp. 246-257, 1984.

[15] T. C. Hou and V. Li, "Transmission Range Control in Multihop Packet Radio Networks", Communications, IEEE Transactions, vol. 34, no. 1, pp.38-44, 1986.

[16] B. Karp and H. T. Kung, "GPSR: Greedy Perimeter Stateless Routing for Wireless Networks," in MobiCom '00: Proceedings of the 6th annual international conference on Mobile computing and networking. New York, NY, USA: ACM Press, 2000, pp. 243-254.

[17] F. Kuhn, R. Wattenhofer, and A. Zollinger, "An Algorithmic Approach to Geographic Routing in Ad Hoc and Sensor Networks," IEEE/ACM Trans. Netw., vol. 16, no. 1, pp. 51-62, 2008

[18] S. Dulman, M. Rossi, P. Havinga, and M. Zorzi, "On the Hop Count Statistics for Randomly Deployed Wireless Sensor Networks," Int. J. Sen. Netw., vol. 1, no. 1/2, pp. 89-102, 2006.

[19] Yilmaz O., Demirci S., Kaymak Y., Ergun S., \& Yildirim A. (2012)"Shortest hop multipath algorithm for wireless sensor networks". Computer Communications.63, 48-59.

[20] Moser C., Thiele L., Brunelli D., \& Benini L., (2010) "Adaptive power management for environmentally powered systems". IEEE Transactions on Computers 59, 478-491.

[21] Alsalih W., Hassanein H., \& Akl S.(2010).’Placement of multiple mobile data collectors in wireless sensor networks” .Ad Hoc Networks 8, 378-390 